\title{
Correction to: Sarilumab plus methotrexate in patients with active rheumatoid arthritis and inadequate response to methotrexate: results of a randomized, placebo-controlled phase III trial in Japan
}

Yoshiya Tanaka ${ }^{1 *}$, Kazuteru Wada ${ }^{2}$, Yoshinori Takahashi ${ }^{2}$, Owen Hagino ${ }^{3}$, Hubert van Hoogstraten ${ }^{4}$, Neil M. H. Graham ${ }^{5}$ and Hideto Kameda ${ }^{6}$

\section{Correction to: Arthritis Res Ther https://doi.org/10.1186/s13075-019-1856-4}

Following publication of the original article [1], the authors reported an error in Table 2. The data for 'HAQ-DI response (MCID $\geq 0.3$ ), $\mathrm{n}(\%) /$ At week 52' for the 'Sarilumab 150 mg q2w + MTX' and 'Sarilumab 200 mg q2w + MTX' groups should be 41 (50.6) and 37 (46.3), respectively (last row, last two entries of the table).

The corrected table is given below.

\begin{abstract}
Author details
${ }^{1}$ The First Department of Internal Medicine, School of Medicine, University of Occupational and Environmental Health, Japan, 1-1 Iseigaoka,

Yahata-nishi-ku, Kitakyushu 807-8555, Japan. ${ }^{2}$ Sanofi K.K, Opera City Tower 3-20-2 Nishi-Shinjuku, Shinjuku-ku, Tokyo 163-1488, Japan. ${ }^{3}$ Sanofi, 55 Corporate Drive, Bridgewater, NJ 08807, USA. ${ }^{4}$ Sanofi-Genzyme, 500 Kendall St, Cambridge, MA 02142, USA. ${ }^{5}$ Regeneron Pharmaceuticals, Inc., 777 Old Saw Mill River Road, Tarrytown, NY 10591, USA. 'Division of Rheumatology, Department of Internal Medicine, School of Medicine, Toho University, 2-22-36 Ohashi, Meguro-ku, Tokyo 153-8515, Japan.
\end{abstract}

Received: 2 April 2019 Accepted: 2 April 2019

Published online: 16 April 2019

\section{Reference}

1. Tanaka, et al. Sarilumab plus methotrexate in patients with active

rheumatoid arthritis and inadequate response to methotrexate: results of a randomized, placebo-controlled phase III trial in Japan. Arthritis Res Ther. 2019;21:79. https://doi.org/10.1186/s13075-019-1856-4.

\footnotetext{
* Correspondence: tanaka@med.uoeh-u.ac.jp

${ }^{1}$ The First Department of Internal Medicine, School of Medicine, University of Occupational and Environmental Health, Japan, 1-1 Iseigaoka,

Yahata-nishi-ku, Kitakyushu 807-8555, Japan

Full list of author information is available at the end of the article
}

(c) The Author(s). 2019 Open Access This article is distributed under the terms of the Creative Commons Attribution 4.0 International License (http://creativecommons.org/licenses/by/4.0/), which permits unrestricted use, distribution, and reproduction in any medium, provided you give appropriate credit to the original author(s) and the source, provide a link to the Creative Commons license, and indicate if changes were made. The Creative Commons Public Domain Dedication waiver (http://creativecommons.org/publicdomain/zero/1.0/) applies to the data made available in this article, unless otherwise stated. 
Table 2 Efficacy results (mITT population)

\begin{tabular}{|c|c|c|c|c|}
\hline & \multicolumn{4}{|l|}{ Sarilumab } \\
\hline & $\begin{array}{l}\text { Placebo to } 150 \mathrm{mg} \text { q2W }+ \text { MTX } \\
(n=41(n=14 \text { at week } 52))^{\mathrm{a}}\end{array}$ & $\begin{array}{l}\text { Placebo to } 200 \mathrm{mg} \mathrm{q2w}+\mathrm{MTX} \\
(n=40(n=15 \text { at week 52)) }\end{array}$ & $\begin{array}{l}150 \mathrm{mg} q 2 \mathrm{w}+\mathrm{MTX} \\
(n=81)\end{array}$ & $\begin{array}{l}200 \mathrm{mg} q 2 \mathrm{w}+\mathrm{MTX} \\
(n=80)\end{array}$ \\
\hline \multicolumn{5}{|l|}{ Signs and symptoms } \\
\hline \multicolumn{5}{|l|}{ ACR20 response, $n(\%)$} \\
\hline At week 12 & $15(18.5)$ & & $54(66.7)^{* * *}$ & $52(65.0)^{* * *}$ \\
\hline At week 24 & $12(14.8)$ & & $55(67.9)^{* * *}$ & $46(57.5)^{* * *}$ \\
\hline At week 52 & $9(64.3)$ & $10(66.7)$ & $58(71.6)$ & $48(60.0)$ \\
\hline \multicolumn{5}{|l|}{ ACR50 response, $n(\%)$} \\
\hline At week 12 & $5(6.2)$ & & $22(27.2)^{* * * *}$ & $25(31.3)^{* * *}$ \\
\hline At week 24 & $8(9.9)$ & & $35(43.2)^{* * *}$ & $31(38.8)^{* * *}$ \\
\hline At week 52 & $8(57.1)$ & $10(66.7)$ & $37(45.7)$ & $38(47.5)$ \\
\hline \multicolumn{5}{|l|}{ ACR70 response, $n(\%)$} \\
\hline At week 12 & $1(1.2)$ & & $5(6.2)$ & $15(18.8)^{* * *}$ \\
\hline At week 24 & $3(3.7)$ & & $15(18.5)^{* *}$ & $12(15.0)^{*}$ \\
\hline At week 52 & $4(28.6)$ & $3(20.0)$ & $29(35.8)$ & $22(27.5)$ \\
\hline \multicolumn{5}{|c|}{ ACR components, mean (SD) change from baseline at week 24} \\
\hline Tender joint count & $-9.1(10.2)$ & & $-13.4(9.9)$ & $-12.4(11.3)$ \\
\hline Swollen joint count & $-7.2(6.7)$ & & $-10.6(8.1)$ & $-9.5(9.1)$ \\
\hline Pain VAS & $-22.9(27.7)$ & & $-36.5(23.4)$ & $-30.2(23.3)$ \\
\hline Physician global VAS & $-26.8(18.4)$ & & $-41.8(21.6)$ & $-43.9(19.4)$ \\
\hline Patient global VAS & $-18.3(22.6)$ & & $-32.4(21.0)$ & $-30.6(21.9)$ \\
\hline HAQ-DI & $-0.3(0.4)$ & & $-0.5(0.5)$ & $-0.6(0.5)$ \\
\hline CRP, mg/l & $-1.7(12.2)$ & & $-21.1(19.5)$ & $-21.3(18.0)$ \\
\hline \multicolumn{5}{|c|}{ DAS28-CRP response, mean (SD) change from baseline } \\
\hline At week 12 & $-0.8(1.1)$ & & $-2.3(1.1)^{* * *}$ & $-2.3(1.2)^{* * *}$ \\
\hline At week 24 & $-1.5(1.2)$ & & $-2.8(1.0)^{* * *}$ & $-2.8(1.1)^{* * *}$ \\
\hline At week 52 & $-3.1(1.2)$ & $-2.9(1.2)$ & $-3.2(1.2)$ & $-3.2(1.1)$ \\
\hline \multicolumn{5}{|l|}{ DAS28-CRP $<2.6, n(\%)$} \\
\hline At week 12 & $3(3.7)$ & & $21(25.9)^{* * *}$ & $27(33.8)^{* * *}$ \\
\hline At week 24 & $6(7.4)$ & & $29(35.8)^{* * *}$ & $32(40.0)^{* * *}$ \\
\hline At week 52 & $7(50.0)$ & $9(60.0)$ & $41(50.6)$ & $43(53.8)$ \\
\hline \multicolumn{5}{|c|}{ SDAl, mean (SD) change from baseline } \\
\hline At week 12 & $-8.9(12.0)$ & & $-20.7(11.0)^{* * *}$ & $-18.9(11.6)^{* * *}$ \\
\hline At week 24 & $-16.0(11.6)$ & & $-25.2(11.6)^{* * *}$ & $-23.8(11.3)^{* * *}$ \\
\hline At week 52 & $-29.6(9.9)$ & $-23.4(12.4)$ & $-29.4(13.6)$ & $-26.9(11.5)$ \\
\hline \multicolumn{5}{|l|}{$\mathrm{SDAl} \leq 3.3, n(\%)$} \\
\hline At week 12 & 0 & & $2(2.5)$ & $7(8.8)^{* *}$ \\
\hline At week 24 & $1(1.2)$ & & $5(6.2)$ & $10(12.5)^{* *}$ \\
\hline At week 52 & $2(14.3)$ & $1(6.7)$ & $19(23.5)$ & $18(22.5)$ \\
\hline \multicolumn{5}{|c|}{ CDAl, mean (SD) change from baseline } \\
\hline At week 12 & $-8.7(11.4)$ & & $-18.8(10.6)^{* * *}$ & $-16.8(10.9)^{* * *}$ \\
\hline At week 24 & $-15.7(11.1)$ & & $-23.1(11.2)^{* * * *}$ & $-21.7(10.7)^{* * *}$ \\
\hline At week 52 & $-28.4(9.7)$ & $-21.1(11.4)$ & $-27.2(13.1)$ & $-24.8(10.8)$ \\
\hline
\end{tabular}


Table 2 Efficacy results (mITT population) (Continued)

\begin{tabular}{|c|c|c|c|c|}
\hline & \multicolumn{4}{|l|}{ Sarilumab } \\
\hline & $\begin{array}{l}\text { Placebo to } 150 \mathrm{mg} \text { q2w }+ \text { MTX } \\
(n=41(n=14 \text { at week } 52))^{\mathrm{a}}\end{array}$ & $\begin{array}{l}\text { Placebo to } 200 \mathrm{mg} \text { q2w }+ \text { MTX } \\
\left(n=40(n=15 \text { at week 52) })^{\mathrm{a}}\right.\end{array}$ & $\begin{array}{l}150 \mathrm{mg} q 2 w+M T X \\
(n=81)\end{array}$ & $\begin{array}{l}200 \mathrm{mg} q 2 \mathrm{w}+\text { MTX } \\
(n=80)\end{array}$ \\
\hline \multicolumn{5}{|c|}{ CDAI $\leq 2.8, n(\%)$} \\
\hline At week 12 & 0 & & $1(1.2)$ & $5(6.3)^{*}$ \\
\hline At week 24 & $1(1.2)$ & & $5(6.2)$ & $8(10.0)^{*}$ \\
\hline At week 52 & $1(7.1)$ & 0 & $17(21.0)$ & $15(18.8)$ \\
\hline \multicolumn{5}{|l|}{ Physical function } \\
\hline \multicolumn{5}{|c|}{ HAQ-DI, mean (SD) change from baseline } \\
\hline At week 12 & $-0.1(0.3)$ & & $-0.4(0.5)^{* * *}$ & $-0.4(0.5)^{* * *}$ \\
\hline At week 24 & $-0.3(0.4)$ & & $-0.5(0.5)^{* * *}$ & $-0.6(0.5)^{* * *}$ \\
\hline At week 52 & $-0.7(0.6)$ & $-0.5(0.3)$ & $-0.6(0.6)$ & $-0.6(0.6)$ \\
\hline \multicolumn{5}{|c|}{ HAQ-DI response (MCID $\geq 0.3), n(\%)$} \\
\hline At week 12 & $19(23.5)$ & & $39(48.1)^{* *}$ & $38(47.5)^{* *}$ \\
\hline At week 16 & $19(23.5)$ & & $37(45.7)^{* *}$ & $37(46.3)^{* *}$ \\
\hline At week 24 & $10(12.3)$ & & $39(48.1)^{* * *}$ & $39(48.8)^{* * *}$ \\
\hline At week 52 & $9(64.3)$ & $8(53.3)$ & $41(50.6)$ & $37(46.3)$ \\
\hline
\end{tabular}

${ }^{*} p<0.05 ;{ }^{* *} p<0.01 ;{ }^{* * *} p<0.001$

${ }^{a}$ Data for combined placebo groups ( $n=81$ ) shown at weeks 12, 16 and 24. ACR American College of Rheumatology, ACR20/50/70 American College of

Rheumatology 20\%/50\%/70\% improvement criteria, CDAI Clinical Disease Activity Index, CRP C-reactive protein, DAS28 Disease Activity Score 28-joint count, HAQDI Health Assessment Questionnaire-Disability Index, MCID minimum clinically important difference, $\mathrm{m} / T$ modified intent-to-treat, $M T X$ methotrexate, $q 2 w$ every 2 weeks, SDAI Simplified Disease Activity Index, SD standard deviation, SJC swollen joint count, TJC tender joint count, VAS visual analog scale 